\title{
Performance Modelling of IEEE 802.11g Wireless LAN
}

\author{
Choman Othman Abdullah \\ School of Science Education \\ University of Sulaimani, KRG, Iraq \\ choman.abdullah@univsul.edu.iq
}

\author{
Nigel Thomas \\ School of Computing Science \\ Newcastle University, UK \\ nigel.thomas@ncl.ac.uk
}

\begin{abstract}
Fairness of wireless channel access in terms of utilisation and throughput is studied for IEEE $802.11 \mathrm{~g}$ by using the process algebra PEPA. Three models are presented to describe different operating scenarios. Results are derived which demonstrate when and how unfairness might occur, leading to the penalisation of some nodes in a network.
\end{abstract}

\section{CCS Concepts}

-Networks $\rightarrow$ Network performance modeling;

\section{Keywords}

WLAN, IEEE 802.11g, Performance modelling, Fairness, PEPA.

\section{INTRODUCTION}

Understanding the performance characteristics of wireless networks is vital in order to obtain efficient and effective deployments. In this paper we focus on evaluating the fairness of channel access due to topographic effects in the layout of communicating nodes under IEEE $802.11 \mathrm{~g}$. If a deployment gives rise to unfairness then the performance obtained by the affected nodes may be significantly below that which is expected when there is competition for channel access, which may cause significant issues for individuals or service delivery.

IEEE 802.11 [1] is the most established and widespread class of wireless network protocol currently available. IEEE 802.11 is categorised as a set of protocols, $802.11 \mathrm{a} / \mathrm{b} / \mathrm{g} / \mathrm{n} / \mathrm{ac}$, with similar structure, but different operating ranges (power, data rate, message length, etc). Many researchers have analysed IEEE 802.11 protocols in terms of the rate adaptation scheme, performance of IEEE 802.11 MAC layer and different performance metrics. For instance, Zhai et al [21]

* Choman O. Abdullah is a PhD student at Newcastle University, UK, c.o.a.abdullah@ncl.ac.uk. have attempted to "characterize the probability distribution model of the MAC layer packet service time", based on deriving the probability mass function of the inter-departure interval to understand delay mean and throughput at several traffic loads. In [17] Shehadeh and Chasaki explained that to access the medium for any devices the capability and fairness are the most important issue and are essential for reaching great effectiveness in numerous wireless devices and wireless traffics. Additionally, users expect to access the medium in wireless networks fairly for resources in public areas.

In this paper we extend a previous model for $802.11 \mathrm{~b}$ to consider various deployment scenarios in $802.11 \mathrm{~g}$. The two versions of the protocol have extensive similarities, but differ in terms of transmission speeds, rates, ranges and delays. As such, we have been able to adapt a model from [11], which we had previously studied in [2], to consider the fairness of three deployment scenarios in $802.11 \mathrm{~g}$. The paper is organized as follows: Section 2 presents a background and related work to generally review IEEE 802.11 and PEPA. The model for each scenario is specified using PEPA and discussed in Section 3. The parameters are describes in Section 4. The results and figures are given in Section 5. Finally, conclusion and future works are provided in Section 6.

\section{BACKGROUND AND RELATED WORK}

\subsection{IEEE 802.11}

With low cost and higher speed the 802.11 protocols have been commonly used as a basic standard for WLANs [1]. They are categorised to different protocols, such as $\mathbf{a} / \mathbf{b} / \mathbf{g} / \mathbf{n}$ and ac. Some research studied the throughput and fairness in $802.11 \mathrm{~b}$ multi-rate with a COT-based algorithm. Zhang et $a l$ in [22] argued that by amending a packet size, the wireless stations can reach COT-Fairness as it can reach to high performance. Moreover, the packet size, transmission rate, distance between stations, retry limit and network topology are considered, as they affect the performance of IEEE 802.11b, as Pham et al have studied in their experiments [15]. However, Pham et al have only studied transmission between two stations and have not extended the number of nodes in their experiments, and have not applied their approach to other protocols, like $802.11 \mathrm{~g}$.

Razafindralambo and Valois [16] have explored a symmetric hidden terminal scenario. They have examined a scenario of three transmitting node pairs under four backoff algorithms. To understand the performance of backoff algorithms in multi-hop ad hoc networks, they have evaluated 
the performance of each backoff algorithm for efficiency and when possible from a fairness point of view. However, they did not consider the retry limit, reducing and increasing the process to optimise for fairness performance metrics. Others have considered both short-term and long-term fairness on $802.11 \mathrm{~b}$ and the performance evaluation of fairness for accessing channel [11] for communicating pairs in terms of medium utilization and throughput.

Theoretical analysis method has been used by Lee [13] to measure error-free and errorprone wireless channels with the higher transmission rates under $802.11 \mathrm{~g}$. Lee studied the capacity throughput performance, as he argued that when the speed of mobile of a station is increased, the throughput is reduced. However, he did not consider utilisation, and did not argue for other scenarios with numerous nodes.

The maximum rate of $802.11 \mathrm{~g}$ is $54 \mathrm{Mbps}$, which has the same rate of 802.11a. However, 802.11g is more compatible with 802.11 b as they both use DSSS in many WLAN devices. Ming [9] concentrated on WLAN throughput performance, as he has argued that the best 802.11g OFDM throughput performance can be obtained in specific slow time. Additionally, Kanduri et al in [10] have studied the structures of IEEE $802.11 \mathrm{~g}$ in maximum data rate WLANs, as it might increase WLAN requests by users. Vucinic et al in [19] have studied performance degradation for $802.11 \mathrm{~g}$ in terms of access delay for dissimilar nodes and throughput, as they have analysed collision probability, channel access delay and channel throughput in associate with the AP in backwards compatibility. In this research we have studied the performance of IEEE $802.11 \mathrm{~g}$.

\subsection{Network protocol analysis with PEPA}

Many simulations can be used to analyse models of WLAN environments. While simulations can support a detailed representation of protocol actions, the approach may suffer from excessively long run times, making parameter optimisation infeasible. A typical solution to this problem is to employ some form of stochastic modelling technique (see for example [6] and [14]) to create an abstract representation of the system which can solve analytically or numerically to derive measures of interest, which can then be verified using simulation as necessary. Both simulation and mathematical modelling can suffer from problems of lack of behavioural insight and lack of modelling reusability. Formal modelling techniques, such as stochastic Petri nets, stochastic automata and stochastic process algebra, seek to overcome these issues by providing a high level modelling paradigm, which can be used to the model behaviour and to derive numerical solutions and to predict performance.

PEPA [8] is a process algebra which provides a useful modelling formalism for investigating properties of protocols and other well defined systems. PEPA models are specified in terms of components which interact through shared actions. In PEPA actions have a duration, which is determined by a rate parameter of the negative exponential distribution. It is shared actions, where a rate may be given by one or both interacting components, is determined by the slowest participant. In network protocol terms, components can be network nodes and the transmission media and shared actions can be thought of as the transmission of messages (packets) from one node to another through the medium. The combination of all components into a single system gives rise to labelled transition system where the transitions between states are negative exponentially distributed actions, hence the resultant system is a continuous time Markov chain (CTMC). The PEPA Eclipse Plug-in tool [6] supports a range of powerful analysis techniques for Markov Process (CTMC), systems of ordinary differential equations (ODE) and stochastic simulation which allows modellers to derive results (both transient and steady state, with relative ease).

Despite the benefits of using PEPA to model and analyse protocols, there are very few examples in the literature where PEPA has been used to study IEEE 802.11. ArgentKatwala et al [3] studied WLAN protocols and performance models of the 802.11 in terms of its QoS based on PEPA. They argued that most of the technologies have been developed to enhance the reliability of computer networks. In wireless communication protocols security is mandated needs in exchanging data, which must be delivered within a specific time. Moreover, they used PEPA to find properties which cannot be easy to find manually in term of computing quantitative, passage time and increase higher probability for performance demands.

Sridhar and Ciobanu [18] focused on DCP within IEEE 802.11. They described a quantitative analysis of a handoff mechanism and channel mobility. They assessed the handoff channels using $\pi$-calculus, where data can be passed by allowed channels. Kloul and Valois [11] studied performance analysis of the $802.11 \mathrm{~b}$ by using PEPA. Specifically, they investigated an unfairness scenario in MANET. They were interested in system behaviour to measure and investigate the performance of $802.11 \mathrm{~b}$ protocol with different scenarios. We have studied $802.11 \mathrm{~b}$ with two and three pairs scenarios in our former paper [2] using the same approach as [11].

\section{THE MODEL}

\subsection{Basic access mechanism}

Medium access timing in 802.11 generally cooperates using Point Coordination Function $(P C F)$ and Distributed Coordination Functon $(D C F)$. $P C F$ needs a central control object and $D C F$ is based on CSMA/CA. The basic access mechanism (BSA) in 802.11 is $D C F$, which is a common technique used up to $802.11 \mathrm{~g}$. In WLAN to access the medium, the node listens to the channel, when the medium is free to use with no congestion, then it can make its transaction successfully. On successful receipt, the receiving node will transmit an acknowledgement $(A C K)$. However, if two nodes attempt to transmit simultaneously, then collision occurs resulting in an unsuccessful transmission and the initiation of the backoff algorithm. In backoff, an unsuccessful node waits for a random time (backoff period) in the range $[0, C W]$, where $C W$ is a contention window based on the number of transmission failures. The initial value of $C W$ is [31] and [15] for $802.11 \mathrm{~b} / \mathrm{g}$ respectively, and $C W$ is doubled after every unsuccessful transmission, until it reaches to the maximum number [1023], (see [5,7] and $[9,10,20]$ for detailed explorations of the backoff algorithm). $C W$ returns to the initial value after each $A C K$ revived. The backoff technique preferably correlates with collision avoidance, if the channel is occupied the backoff is set with a slot of time $(20 \mu s)$ and after each frame transmission, in case of collision, they required Inter-Frame Space (IFS) is applied. The minimum shortest interval of time is called Short-IFS $(10 \mu s)$ see [12]. The channel activity can monitor by nodes, when the backoff has been generated. If the channel is idle during a adequate 
long time "Distributed-IFS $(50 \mu s)$ then the node decreases the backoff. When the backoff is retune to zero, the packet can transmit. A part from that, if the channel is not free to use, the node monitors the channel till it becomes idle. Then the node decreases its backoff. It is decreasing if the channel is free to use when the node detects a transmission. Latterly of this transmission, when the medium remains idle throughout a DIFS, the decrementation starts again. In effect, during the backoff decrementation, if the node detects a signal but, because of any congestion, not a transmission in progress, the node practises an Extended-IFS (364 $\mu s)$ instead of DIFS. After a successful transmission, an $A C K$ will receive after SIFS. See Table 1 and Figure 1.

\begin{tabular}{|l|l|l|}
\hline \multirow{2}{*}{ Attribute } & \multicolumn{2}{c|}{ Typical value } \\
\cline { 2 - 3 } & \multicolumn{1}{|c|}{$\mathbf{8 0 2 . 1 1 b}$} & \multicolumn{1}{c|}{$\mathbf{8 0 2 . 1 1} \mathrm{g}$} \\
\hline CWmin, and CWmax & 31, and 1023 & $15(\mathrm{pure})$, and 1023 \\
\hline Slot time & $20 \mu \mathrm{s}$ & $20 \mu \mathrm{s}, 9 \mu \mathrm{s}$ \\
\hline SIFS & $10 \mu \mathrm{s}$ & $10 \mu \mathrm{s}$ \\
\hline DIFS & $50 \mu \mathrm{s}$ & $50 \mu \mathrm{s}, 28 \mu \mathrm{s}$ \\
\hline EIFS & $364 \mu \mathrm{s}$ & $364 \mu \mathrm{s}$ \\
\hline
\end{tabular}

Table 1: Attribute values of $802.11 \mathrm{~b} / \mathrm{g}$

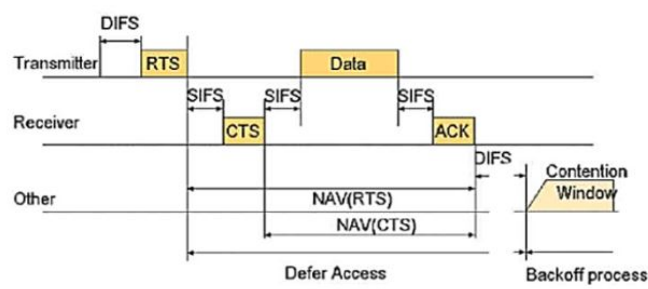

Figure 1: RTC-CTS and Data-ACK scheme.

\subsection{Scenarios modelled with PEPA}

This section presents the modelling of two, three and four pairs scenarios with PEPA. The pair components have identical behaviour in each case, but the medium is different in each scenario.

Note 1: In these scenarios all pairs have the same behaviour, they all behave the same as pairs $\mathbf{A}$ as shown in Scenario 1.

Note 2: In the model of two and three pairs scenarios the mediums are the same, but with different cooperation sets. Note 3: In these scenarios the pairs are consistently trying to transmit therefore each of them is attempting to find the maximum throughput.

\subsubsection{The two pairs scenario (Scenario 1)}

This scenario has three components, pair A, pair B (both are symmetric), and the medium component as denoted by Pair_A, Pair_B and Med_F (see Figure 2). The pairs do not interact directly with each other, bur both interact with the medium, which affects their behaviour. Once, one node in a pair attempts to transmit, its partner node waits to receive an $A C K$. Pair_A and Pair_B are equally occupying the channel (Medium_F). To transmit, Pair_A draws a backoff and becomes Pair_A0, then Pair_AO starts to count the DIFS to become Pair_A1 or stays in the queue as Pair_A5. As Pair_A5 it waits before becoming Pair_A4. All SIFS and EIFS will count until the backoff end, then the packet can transmit in Pair_A2 finally the $A C K$ will be received in Pair_A6. Clearly, this scenario is fair, as each pair can access the medium equally.

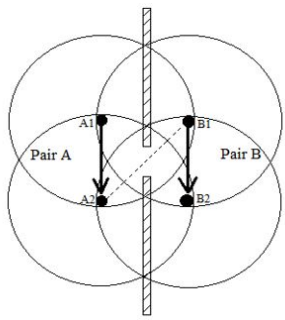

Figure 2: Tow pairs scenario (Scenario 1).

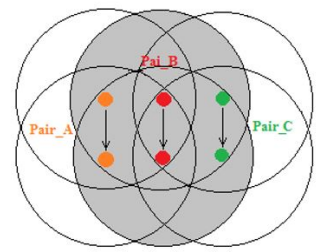

Figure 3: Three pairs scenario (Scenario 2).

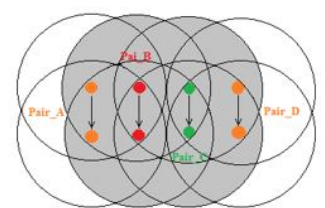

Figure 4: Four pairs scenario (Scenario 3).

\begin{tabular}{|c|c|c|}
\hline Pair_A & $\stackrel{\text { def }}{=}$ & $($ draw_backoff, $r)$.Pair_AO \\
\hline Pair_AO & $\begin{array}{l}\stackrel{\text { def }}{=} \\
+\end{array}$ & $\begin{array}{l}\text { (count_difs } A, \mu \text { difs }) . P a i r \_A 1 \\
(\text { queueA, } \top) . P a i r \_A 5\end{array}$ \\
\hline Pair_A1 & $\begin{array}{l}\stackrel{\text { def }}{=} \\
+ \\
+\end{array}$ & 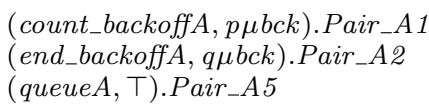 \\
\hline Pair_A2 & $\begin{array}{l}\stackrel{\text { def }}{=} \\
+\end{array}$ & $\begin{array}{l}(\text { transmit } A, \mu \text { data }) . P a i r \_A 3 \\
(\text { queue } A, \top) . P a i r \_A 5\end{array}$ \\
\hline Pair_A3 & $\stackrel{\text { def }}{=}$ & $($ count_sifs, $\mu$ sifs $) . P a i r \_A 6$ \\
\hline Pair_A4 & $\begin{array}{l}\stackrel{\text { def }}{=} \\
+ \\
+\end{array}$ & 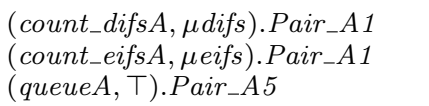 \\
\hline Pair_A5 & $\stackrel{\text { def }}{=}$ & $($ wait,$\mu$ data $) . P a i r_{-} A 4$ \\
\hline Pair_A6 & $\stackrel{\text { def }}{=}$ & $($ ackA, $\mu a c k) . P a i r \_A$ \\
\hline
\end{tabular}

Component (Med_F): This component is used by pair A and B. When a specific pair starts to occupy the medium then the other one are stops to transmit, as follows:

$$
\text { Scenario1 } \stackrel{\text { def }}{=}\left(\left(P a i r_{-} A \bigotimes_{\mathcal{K}} M e d_{-} F\right) \bigotimes P a i r_{-} B\right.
$$

Where the sets $\mathcal{K}$ and $\mathcal{L}$ are:

$\mathcal{K}=\{$ transmit $A$, ack $A$, queueA, count_difs $A$, count_backoff $A$, end_backoff $A$, count_eifs $A\}$. $\mathcal{L}=\{$ transmit $B$, ack $B$, queue $B$, count_difs $B$, count_backoff $B$, end_backoff $B$, count_eifs $B\}$.

$$
\begin{aligned}
& M e d_{-} F \stackrel{\text { def }}{=}(\text { transmitA, } \top) . M e d_{-} F 2 \\
& +\quad \text { (transmitB, } \top) . M e d \_F 1 \\
& +\quad \text { (count_difsA, T).Med_F } \\
& +\quad \text { (count_backoffA, T).Med_F } \\
& +\quad(\text { end_backoff } A, \top) \cdot M e d_{-} F \\
& +\quad \text { (count_eifsA, } \top) \text {.Med_F } \\
& +\quad \text { (count_difsB, T).Med_F } \\
& +\quad \text { (count_backoffB, } \top) \text {.Med_F }
\end{aligned}
$$




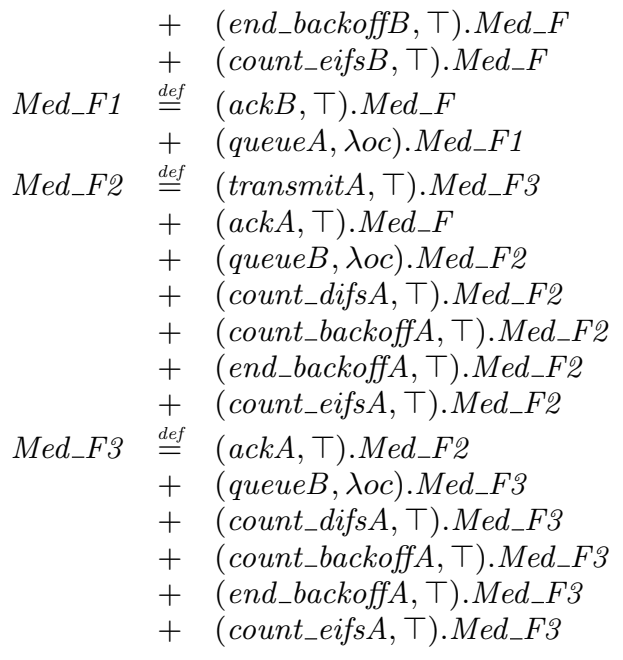

\subsubsection{The three pairs scenario (Scenario 2)}

$(\mathbf{A}, \mathbf{B}, \mathbf{C}$ and $\mathbf{F})$ as they are denoted by (Pair_A, Pair_B, Pair_C and Med_F) have been used in this scenario. External pairs (A and $\mathbf{C}$ ) are symmetric and cannot hear each other, but the central pair (B) can hear the others. Once any external pair is communicating then the central one is queueing, till the channel is free to use. Consequently, the central has less chance to access the medium. Thus, this scenario demonstrates unfairness. The central pair will be unfairly disadvantaged as it will be out competed by externals, as it has to wait until both external pairs are idle, whereas the externals can transmit simultaneously.

PEPA model: All pairs with the medium are occupied by one or both externals or the central. AS follows :

$$
\text { Scenario2 } \left.\stackrel{\text { def }}{=}\left(\underset{\mathcal{L}}{\left(P_{\text {Pair_}} A \| P a i r_{-} C\right.}\right) \bigotimes_{\mathcal{K}} M e d \_F\right)
$$

where $\mathcal{K}=\{$ transmit, ack, queue, count_difs, count_backoff, end_backoff, count_eifs $\}$.

$\mathcal{L}=\{$ transmit, ack $B$, queue $B$, count_difs $B$ count_backoff $B$, end_backof $f B$, count_eifs $B\}$.

\subsubsection{The four pairs scenario: Scenario 3}

Scenario 3 has two central and two external pairs (see Figure 3). There are five components in the model, which are Pair_A, Pair_B, Pair_C and Pair_D) and Medium_F. $P a i r_{-} A$ and $P a i r_{-} D$ are external pairs, independent of each other and symmetric. Pair_B and Pair_C) are central pairs. If $A$ is transmitting then $B$ is blocked. Similarly, if $D$ is transmitting then $C$ is blocked (and vice versa). Similarly $B$ and $C$ cannot transmit simultaneously. We can understand that both central pairs have less chance to access the medium compared with the external pairs. Hence the situation is not a completely fair, because $\mathbf{B}$ and $\mathbf{C}$ are penalised, however it is not as restrictive as the three pairs scenario, as if $\mathbf{A}$ is transmitting (but not $D$ ) then $\mathbf{C}$ still has a chance to access the channel. Similarly, if $\mathbf{D}$ is transmitting (but not $A$ ) then $\mathbf{B}$ has a chance to access the channel. By this approach in comparison to three pairs scenario is relatively fair scenario when compared with the three pairs scenario.

PEPA model of Scenario 3: The medium has collaborate with all pairs as they are symmetric. Either it is oc- cupied by any externals or central pairs. Component A can communicate with $\mathbf{B}$ through the medium $\mathbf{F}$, but both externals cannot interact with each other, this scenario is cooperated between all components that are defined as:

$$
\text { Scenario3 } \stackrel{\text { def }}{=}(\text { Pair_A } \| \text { Pair_B } \| \text { Pair_C } \| \text { Pair_D })
$$

$\mathcal{L}=\left\{\right.$ transmit $A$, ack $A$, queueA, count_difs $A$, count $_{b}$ ackoff $A$, end_backoff $A$, count_eifs $A$, transmit $B$, ack $B$, queueB, count_difsB, count_backoff $\mathrm{f}$, end_backof $f B$, count_eifsB,

\begin{tabular}{|c|c|}
\hline $\begin{array}{l}F \stackrel{d e}{=} \\
+ \\
+ \\
+ \\
+ \\
+ \\
+ \\
+ \\
+ \\
+ \\
+ \\
+ \\
+ \\
+ \\
+ \\
+ \\
+ \\
+ \\
+\end{array}$ & 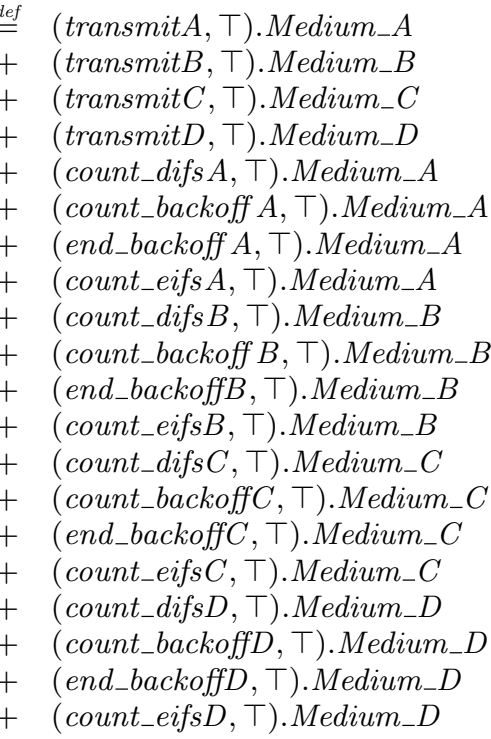 \\
\hline Medium_A & $\begin{array}{l}\stackrel{\text { def }}{=} \quad(\text { transmit } C, \top) \cdot M e d i u m_{-} A C \\
+\quad(\text { transmit } D, \top) \cdot M e d i u m_{-} A D \\
+\quad(\text { ack } A, \top) \cdot M e d i u m_{-} F \\
+\quad(\text { queue } B, \lambda o c) \cdot M e d i u m \_A\end{array}$ \\
\hline Medium_D & $\begin{array}{ll}\stackrel{\text { def }}{=} & (\text { transmit } A, \top) \cdot M e d i u m_{-} A D \\
+ & (\text { transmit } B, \top) \cdot M e d i u m \_B D \\
+ & (\text { ackD }, \top) \cdot M e d i u m_{-} F \\
+ & (\text { queue } C, \lambda o c) \cdot M e d i u m_{-} D\end{array}$ \\
\hline Medium_B & $\begin{array}{l}\stackrel{\text { def }}{=} \quad(\text { transmitD }, \top) \cdot M e d i u m_{-} B D \\
+\quad(\text { ackB }, \top) \cdot M e d i u m_{-} F \\
+\quad(\text { queue } A, \lambda o c) \cdot M e d i u m_{-} B \\
+\quad(\text { queue } C, \lambda o c) \cdot M e d i u m_{-} B\end{array}$ \\
\hline Medium_C & $\begin{array}{l}\stackrel{\text { def }}{=}(\text { transmit } A, \top) \cdot M e d i u m_{-} A C \\
+\quad(\text { ack } C, \top) \cdot M e d i u m_{-} F \\
+\quad(\text { queue } B, \lambda o c) \cdot M e d i u m_{-} C \\
+\quad(\text { queueD }, \lambda o c) \cdot M e d i u m_{C}\end{array}$ \\
\hline Medium_AD & $\begin{array}{l}\stackrel{\text { def }}{=} \quad(\text { ack } A, \top) \cdot \text { Medium_D } \\
+\quad(\text { ackD }, \top) \cdot \text { Medium_A } \\
+\quad(\text { queueB }, \lambda o c) \cdot \text { Medium_AD } \\
+\quad(\text { queue } C, \lambda o c) . \text { Medium_AD }\end{array}$ \\
\hline Medium_BD & 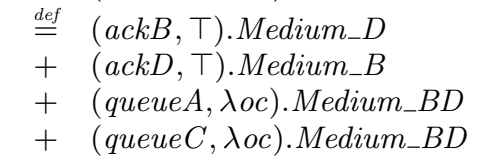 \\
\hline Medium_AC & $\begin{array}{l}\stackrel{\text { def }}{=} \quad(\text { ack } A, \top) \cdot \text { Medium_C } \\
+\quad(\text { ack } C, \top) \cdot \text { Medium_A } \\
+\quad(\text { queueB }, \lambda o c) \cdot \text { Medium_AC } \\
+\quad(\text { queue } C, \lambda o c) . \text { Medium_AC }\end{array}$ \\
\hline
\end{tabular}
transmitC, ackC, queueC, count_difs $C$, count_backoffC, end_backoffC, count_eifs $C$, transmit $D$, ackD, queueD, count_difs $D$, count_backoff $D$, end_backof $f D$, count_eifs $D\}$ 


\section{PARAMETERS}

IEEE 802.11 standard has very specific interframe spacing, which coordinates access to the medium for transmitting frames. For convenience, in this study $\mathbf{p}$ and $\mathbf{q}(\mathbf{q}=1-\mathrm{p})$ are equal to 0.5. According to the IEEE $802.11 \mathrm{~g}$ definition and PHY standards, the data rate per stream are $(6,9,12,18$, 24, 36, 48, and 54) Mbit/s [5] and [10]. These rates have been applied with packet payload size $(700,900,1000,1200$, 1400 and 1500) bytes and, the packets per time unit for arrival and departure rate are $\lambda o c=100000$ and $\mu=200000$ respectively. In this model ( $\mu a c k$ ) shows as a rate of $A C K$ of packages, $\mu a c k=$ Channel throughput $/$ (Ack length $=1$ byte). Also, $\mu$ data is a rate of waiting action for packages, it is calculated by channel throughput/Packet payload, after multiplying with $10^{-6}$ it changes to bytes per second. As, WLAN is used the CSMA/CA, (IFS, $C W$ and $A C K$ ) techniques.

\subsection{Inter-Frame Space (IFS)}

802.11 is a large system of timers. Before each frame can transmit, the length of the IFS is depend on the previous frame type, if noise occurs, the required (IFS) is used. Possibly, when transmission of a particular frame ends and before another one starts the IFS applies a delay for the channel to stay clear. It is an essential idle period of time needed to ensure that other nodes may access the channel. The main purpose of an IFS is to supply a waiting time for each frame transmission in a particular node, to allows the transmitted signal to reach another node (essential for listening). 802.11 protocol has several IFS: SIFS, DIFS, EIFS and Slot time, see $[4,5]$ and $[9]$.

\subsubsection{Short Inter-Frame Space (SIFS)}

SIFS is the shortest Inter-Frame time for highest priority transmissions used with DCF, measured by micro seconds. SIFS is important in 802.11 to better process a received frame. It is equal to $10 \mu \mathrm{s}$ in $802.11 \mathrm{~b} / \mathrm{g} / \mathrm{n}$.

\subsubsection{DCF Inter-Frame Space (DIFS)}

$D I F S$ is a medium priority waiting time after SIFS to monitor the medium. If the channel is idle again, the node waits for the DIFS. Usually the DIFS is longer than SIFS. After the node determines that the channel is idle for a specific of time (DIFS) then it waits for another (backoff). DIFS $=$ SIFS $+(2 \times($ Slot time $=20 \mu \mathrm{s}$ in $802.11 \mathrm{~b} / \mathrm{g} / \mathrm{n}))$.

\subsubsection{Extended Inter-Frame Space (EIFS)}

When the node can detect a signal and DIFS is not functioning during collision, the transmission node uses EIFS instead of DIFS, (used with erroneous frame transmission). It is the longest of the IFS, but, it has the lowest priority after DIFS. EIFS (in $D C F$ ) can derive by:

EIFS $=$ SIFS + DIFS + transmission time of Ack frame at lowest basic rate.

\subsection{Contention Window $(\mathrm{CW})$}

After a node has experimental an idle channel with appropriate IFS, the node waits to minimise any collisions (otherwise many waiting nodes might transmit simultaneously). Before sending any frame the node waits a random period. In CSMA/CA this period is called the backoff and is selected by node from a Contention Window $(C W)$. Faster backoff needs less time to wait, so transmission will be faster too, unless there is a collision. Backoff is chosen over $[0, C W]$, however, $\mathrm{CW}=\mathrm{CW}$ min for all station or nodes if a node successfully transmits a packet and then receives an $A C K$.
But in the case of not transmission, the node is dealing another (backoff), then the $C W$ size is increased exponentially until it reaches CWmax. Finally, the CW is reset to CWmin when the packet is received properly. CW and backoff are set as follows:

$\mathrm{CWmin}=15($ for $802.11 \mathrm{~g}), \mathrm{CW} \max =1023$. And CWmin augmented by $2 \mathrm{n}-1$ on each retry.

Backoff Time $=($ Random () $\bmod (\mathrm{CW}+1)) \times$ Slot Time.

If BackoffTimer $=\mathrm{b}$, when $\mathrm{b}$ is a random integer, also CWmin $<\mathrm{b}<\mathrm{CW} \max$

We have used the mean of $\mathrm{CW}$ to calculate:

$\mu$ bck $=10^{6} /$ Mean of CW $\times$ Time Slot. An $A C K$ is sent by the receiver when it gets a packet successfully, it is a precaution action to notify when collisions occur.

$\mu$ ack $=$ Date rate(Bps) $/$ packet payload size.

$\mu$ data can be obtained by $\mu$ ack $\times$ packet payload size.

\section{RESULTS AND FIGURES}

\subsection{Results of the two pairs scenario: Scenario1}

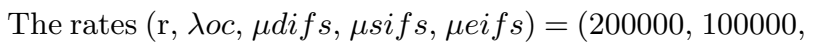
$20000,100000,2747.3)$ are used to measure the utilisation and throughput. Pair $\mathbf{A}$ can receive ACK during its transmitting to $\mathbf{B}$. In this scenario we can understand that it is fairness on each of the medium utilisation and throughput, by using the above parameters and the given formula: Channel utilisation $=P[$ Medium_F $\wedge($ Pair_A2 or Pair_B2 $)]$ $+P\left[\right.$ Medium $\_$F1 $]+P\left[\right.$ Medium $\_$F2 $] \quad$ In Figure 5 the chan-

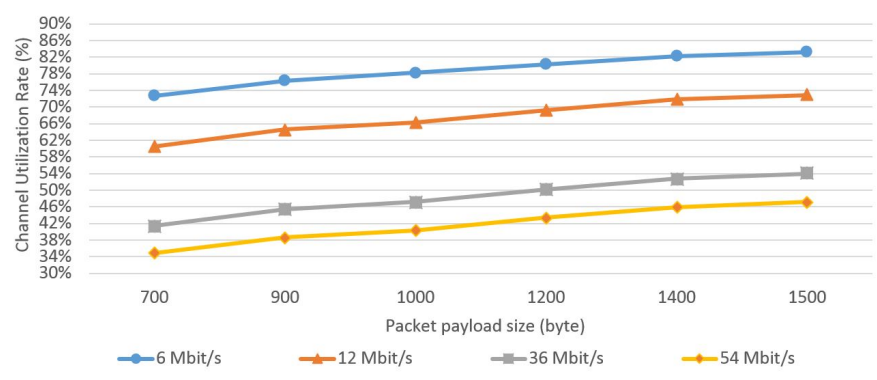

Figure 5: Channel utilization rate in Scenario 1.

nel utilization rate increases as the packet payload size increases, for $6,12,36$, and $54 \mathrm{Mbit} / \mathrm{s}$. This is because the occupied channel time increases as the packet payload size increases (as there is consequently more data to transmit in this model). In $54 \mathrm{Mbit} / \mathrm{s}$ the packet can be sent faster for actual rate transmission. We can see that channel utilization rate in $(6 \mathrm{Mbit} / \mathrm{s})$ is increasing while the packet payload size is increasing for the same speed. Accordingly, it seems that the actual transmission rate will be faster. And, the channel utilisation for each pair is exactly half of the total channel utilisation, hence each pair can access the medium equally. However, the channel throughput decreases when the packet payload size increases. This is because the channel occupancy time is always increasing with increasing the packet payload size from 700 to 1500 bytes, see Figure 6 . Finally, in throughput if we have faster backoff, we need less time to transmit, which means we will obtain faster transition in less time. Once the backoff ends successfully, then each pair can use the medium equally, then the sender receives $A C K$. Hence, we can see that this is a totally fair scenario. 


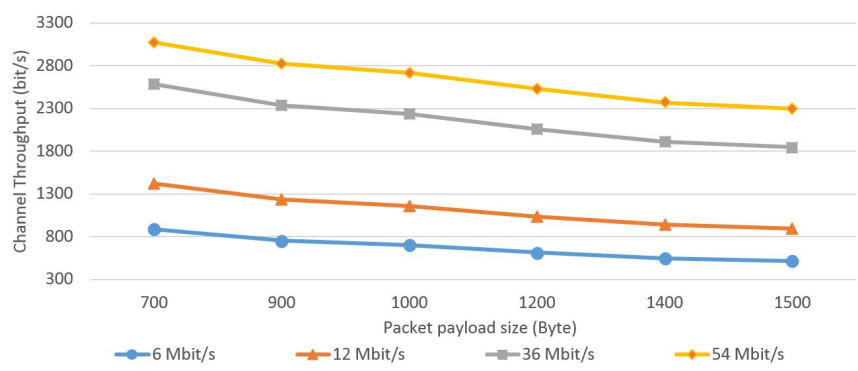

Figure 6: Total throughput for both pairs in Scenario 1.

\subsection{Results of the three pairs scenario: Sce- nario 2}

In Scenario 2 both externals are using the channel equally. However, the behaviour of the central is clearly the same. The channel utilisation for external pairs increases as the packet payload size increases (as in the previous scenario). Both externals are able to transmit at the same time without collisions. As the packet size is increasing at the same time, the external pairs can occupy the channel equally as two symmetric pairs. See Figure 7.

Channel utilisation $=$ Total utilisation $\times$ Throughput A/Total throughput (AckA and AckB)

The channel utilisation of the central pair has a similar

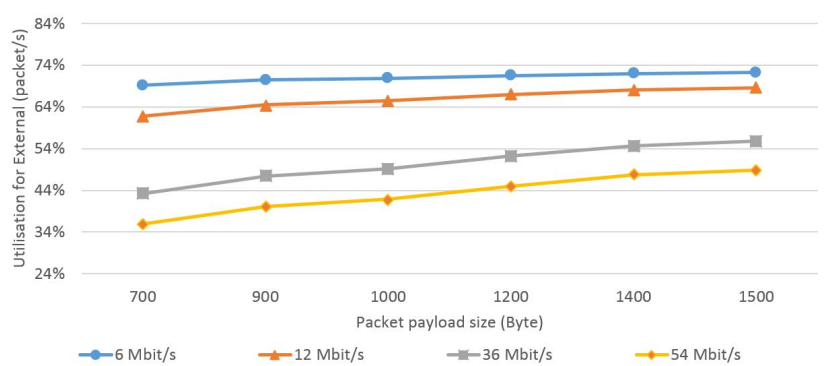

Figure 7: Channel utilisation for externals in Scenario 2.

profile to the externals, but it is much lower because the central has very limited access the channel; most of the time the channel is occupied by the externals (Figure 8).

Channel utilisation $=$ Total utilisation $\times$ Throughput B $/$ Total throughput (AckA and AckB)

As the channel utilisation rate increases we understand that

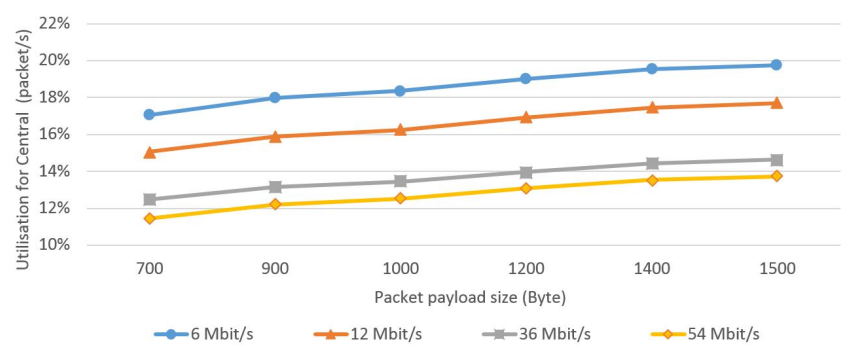

Figure 8: Channel utilisation for the central in Scenario 2.

this scenario is unfair. For the faster transmission we need to increase the packet size or transmit at a lower throughput. Finally, the channel throughput decreases as the packet payload size increases, Figure $\mathbf{9}$ shows externals and Figure 10 central. The faster the channel is in transmitting, then packets will occupy less time in this channel. However, accessing the channel by the central is again limited when compared with the externals. In terms of throughput, this demonstration depicts unfairness; the central is out competed by others and it is unfairly disadvantaged but the externals are unfairly advantaged as each is able to access the channel when the central is blocked.

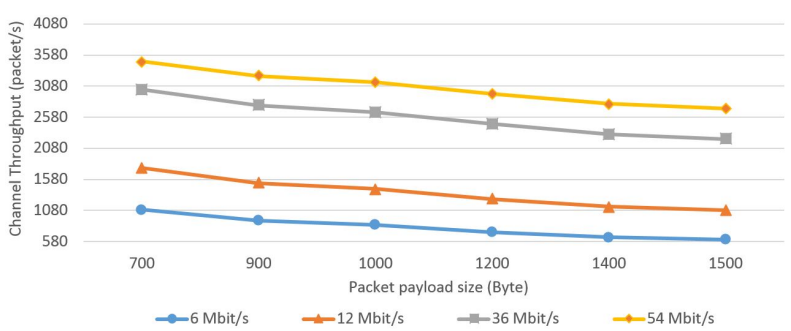

Figure 9: Channel throughput for externals in Scenario 2.

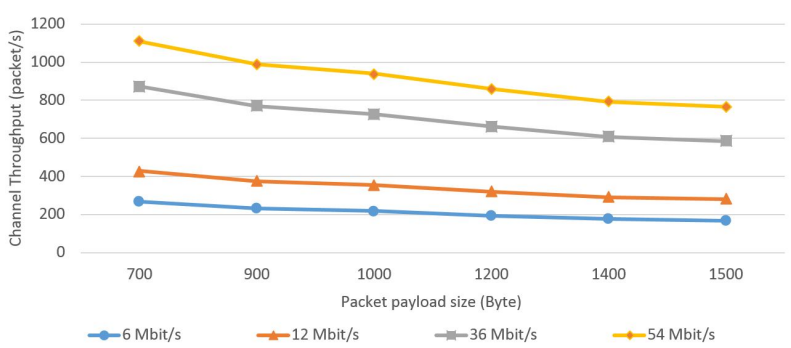

Figure 10: Channel throughput of each central (Scenario 2.)

\subsection{Results of the four pairs scenario: Sce- nario 3}

In this scenario the channel utilisation rate for externals are increasing as the packet payload size are increasing too. Because of the duration of occupant the channel is increasing for $\mathbf{A}$ and $\mathbf{D}$, and they can occupy the channel. Figure 11 shows the channel utilisation rate for externals.

The channel utilisation for central pairs increases as the

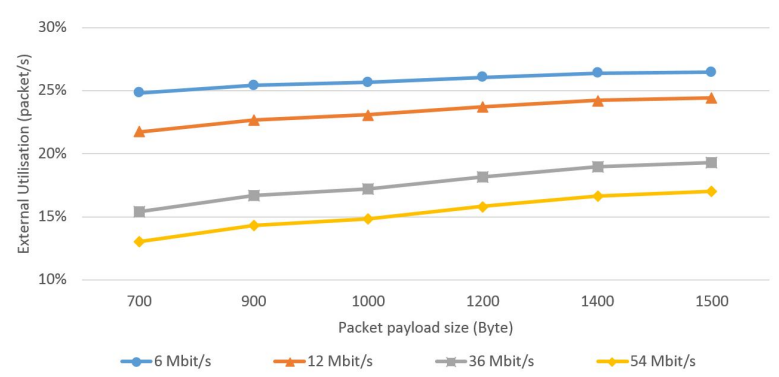

Figure 11: Channel utilisation for externals in Scenario 3.

packet payload size increases too. Each central can use a limited portion of the bandwidth since the medium is occupied by the external transmission most of the time. In this scenario the central pairs cannot use the channel in the same time. Each of them can hear each other and the nearest external neighbour. In Scenario 3 the channel utilisation for each pair is more even compared to the Scenario 2, see Figure 12:

Channel utilisation(externals) $=$ Summation of channel utilisation $+(1-\mathrm{P}[$ Medium $])$.

Channel utilisation $($ central $)=$ Total Utilisation $\times$ Throughput 
B / Total Throughput (AckA, AckB, AckC and AckD).

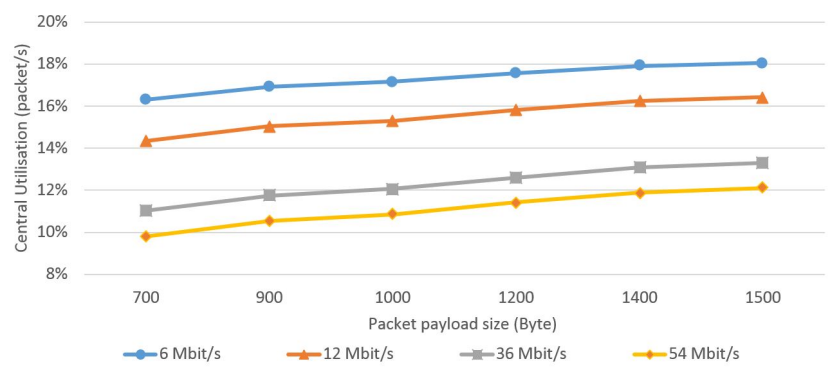

Figure 12: Channel utilisation of each central (Scenario 3.)

In Scenario 3 to analyse the channel utilisation, it is better to calculate it for the external and central pairs separately. The following shows how much time is used in the medium by each central and external pairs.

We have used the following formula for measuring the utilisation for the external in this scenario. The channel utilisation for external is the same as the total channel utilisation. This rate is increasing when the packet payload size is increasing too. Figure 13 shows the utilisation for all pairs. Accordingly, the external pairs occupied the channel equally, also when external pairs are communicated the central pairs are congested. Correspondingly, we calculated the channel utilisation for central pairs separately to better understand the way to access the channel as can be seen in the following. Channel utilisation $($ Externals) $=$ Total Utilisation $\times$ Throughput A/Total Throughput (AckA, AckB, AckC and AckD)

Here, both external pairs and the central pairs occupy the channel equally as they are symmetric.

Finally, channel throughput in Scenario 2 decreases if the

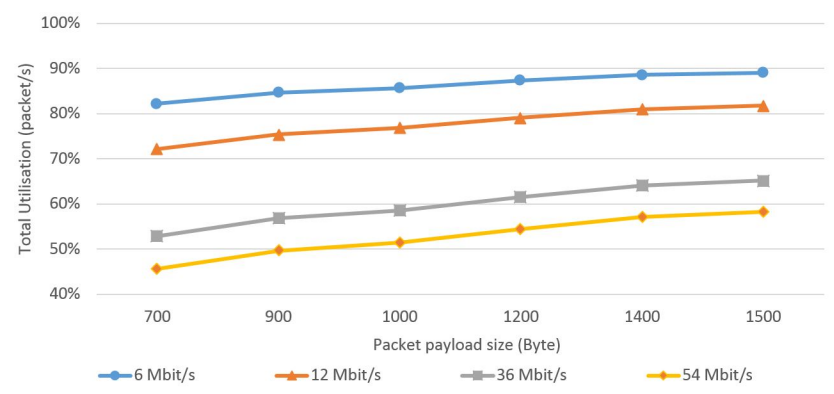

Figure 13: Channel utilisation rate for all pairs in Scenario 3 .

packet payload size increases. In the three node case it is lower because of the channel occupancy time, which means the fastest channel in transmitting packet will occupy less time. In term of throughput this scenario is unfair and is not significant for all pairs. The central pairs are out competed by others and are unfairly disadvantaged, but the externals are unfairly advantaged (Figures 14 and 15).

\section{CONCLUSION}

WLAN is commonly used around the world. Its comfortability and movability have increased its popularity. Many researchers have been studied the performance of WLAN protocols. We concentrated with the performance modelling

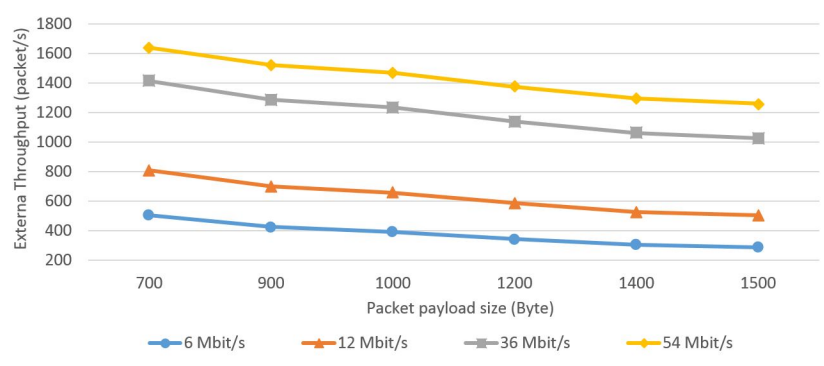

Figure 14: Channel throughput rate for external pairs in Scenario 3.

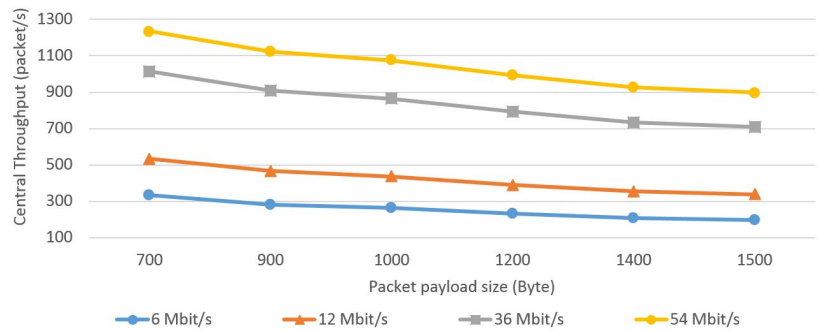

Figure 15: Channel throughput rate for central pairs in Scenario 3 .

of IEEE $802.11 \mathrm{~g}$. We have analysed it by using PEPA for three different scenarios by focusing on CSMA/CA, performance improvement and accessing the medium, in terms of medium utilisation and throughput. Results are derived under heavy load; nodes will attempt to transmit whenever possible. Thus we aim to derive the maximum total throughput and utilisation, but under such conditions we also demonstrate the maximum imbalance in the behaviour of the different pairs.

In Scenario 1 either pair can access the channel whenever it is free and each is blocked when the other is transmitting. Given the inherent symmetry in this scenario the two pairs of nodes have equal access to the medium. However, Scenario $\mathbf{2}$ is unfair because the central pair has less chance to access the channel and cannot compete with the other pairs. The central pair is blocked when either external pair is transmitting. The central pair is therefore disadvantaged whereas the external pairs have an unfair advantage in being able to access the channel even when the other external pair is transmitting. Scenario $\mathbf{3}$ is fairer than Scenario $\mathbf{2}$ as all nodes must compete to gain access and all have the opportunity for access except when their nearest neighbours are transmitting. The central pairs are still disadvantaged compared with the external pairs, although the disparity here is much less than in Scenario 2.

It is clear from the results presented here that the network topology may have a profound effect on the obtained performance for individual nodes, which may deviate considerably from the average. Therefore performance studies which ignore topological effects may be seriously misleading.

In the future, we will expand our research to investigate additional scenarios with other protocols. Future work will explore new models for 802.11n and 802.11ac. We will follow 
tasks that are helpful to understand and redesign new models for $802.11 \mathrm{~g} / \mathrm{n}$ and make a comparison with the current model. A more detailed consideration of different backoff algorithms will also be undertaken to better understand the impact which backoff may have on unfairness.

\section{ACKNOWLEDGEMENTS}

The financial support by Kurdistan Regional Government and Newcastle University is greatly appreciated. The authors wish to thank Leila Kloul for her generous help with early versions of this work.

\section{REFERENCES}

[1] ISO/IEC standard for information technology telecommunications and information exchange between systems - local and metropolitan area networks specific requirements part 11: Wireless LAN medium access control (MAC) and physical layer (PHY) specifications (includes IEEE std 802.11, 1999 edition; IEEE std 802.11a.-1999; IEEE std 802.11b.-1999; IEEE std 802.11b.-1999/cor 1-2001; and IEEE std 802.11d.-2001). ISO/IEC 8802-11 IEEE Std 802.11 Second edition 2005-08-01 ISO/IEC 8802 11:2005(E) IEEE Std 802.11i-2003 Edition, pages 1-721, 2005.

[2] C.O. Abdullah and N. Thomas. Formal performance modelling and analysis of IEEE 802.11 wireless LAN protocols. In UK Performance Engineering Workshop, 2015.

[3] A. Argent-Katwala, J. T Bradley, N. Geisweiller, S.T. Gilmore, and N. Thomas. Modelling tools and techniques for the performance analysis of wireless protocols. Advances in Wireless Networks: Performance Modelling, Analysis and Enhancement, page 3, 2008.

[4] C. Chou, K.G. Shin, and S.N. Shankar. Inter-frame space (IFS) based service differentiation for IEEE 802.11 wireless LANs. In Proceedings of the 58th IEEE Vehicular Technology Conference, volume 3, pages 1412-1416. IEEE, 2003.

[5] A. Duda. Understanding the performance of 802.11 networks. In Proceedings of the 19th International Symposium on Personal, Indoor and Mobile Radio Communications, volume 8, pages 1-6, 2008.

[6] S. Gilmore and J Hillston. The PEPA workbench: A tool to support a process algebra-based approach to performance modelling. In Computer Performance Evaluation Modelling Techniques and Tools, pages 353-368. 1994.

[7] M. Heusse, F. Rousseau, G. Berger-Sabbatel, and A. Duda. Performance anomaly of 802.11b. In Proceedings of the 22nd Annual Joint Conference of the IEEE Computer and Communications Societies, volume 2. IEEE, 2003.

[8] J. Hillston. A compositional approach to performance modelling. Cambridge University Press, 2005.

[9] M.J. Ho, J. Wang, K. Shelby, and H. Haisch. IEEE 802.11g OFDM WLAN throughput performance. In Proceedings of the 58th IEEE Vehicular Technology Conference, volume 4, pages 2252-2256, 2003.

[10] R. Khanduri, S. Rattan, and A. Uniyal. Understanding the features of IEEE $802.11 \mathrm{~g}$ in high data rate wireless LANs. International Journal of Computer Applications, 64(8), 2013.

[11] L. Kloul and F. Valois. Investigating unfairness scenarios in MANET using 802.11b. In Proceedings of the 2nd ACM international workshop on Performance evaluation of wireless ad hoc, sensor, and ubiquitous networks, number 1-8, 2005.

[12] P. Kumar and A. Krishnan. Throughput analysis of the IEEE 802.11 distributed coordination function considering capture effects. International Journal of Automation and Computing, 8:236-243, 2011.

[13] H.C. Lee. A MAC layer throughput over error-free and error-prone channel in the 802.11 a/g-based mobile LAN. In Proceedings of the 9th Malaysia International Conference on Communications, pages 846-851, 2009.

[14] I. Mitrani. Probabilistic modelling. Cambridge University Press, 1998.

[15] D. Pham, Y.A. Sekercioglu, and G.K. Egan. Performance of IEEE 802.11b wireless links: An experimental study. In Proceedings of the IEEE Region 10 Conference (TENCON 2005), 2005.

[16] T. Razafindralambo and F. Valois. Performance evaluation of backoff algorithms in 802.11 ad-hoc networks. In Proceedings of the 3rd ACM international workshop on Performance evaluation of wireless ad hoc, sensor and ubiquitous networks, pages 82-89, 2006.

[17] Y.E.H. Shehadeh and D. Chasaki. Secure and efficient medium access in wireless networks. In Proceedings of the 4 th International Conference on Consumer Electronics, pages 243-244. IEEE, 2014.

[18] KN. Sridhar and G. Ciobanu. Describing IEEE 802.11 wireless mechanisms by using the $\pi$-calculus and performance evaluation process algebra. In Applying Formal Methods: Testing, Performance, and $M / E$-Commerce, pages 233-247. 2004.

[19] M. Vucinic, B. Tourancheau, and A. Duda. Simulation of a backward compatible IEEE 802.11g network: Access delay and throughput performance degradation. In Embedded Computing (MECO), 2012 Mediterranean Conference on, pages 190-195, 2012.

[20] S. Wang, Y. Chen, T. Lee, and A. Helmy. Performance evaluations for hybrid IEEE $802.11 \mathrm{~b}$ and $802.11 \mathrm{~g}$ wireless networks. In Proceedings of the 24th IEEE International Performance, Computing, and Communications Conference, 2005.

[21] H. Zhai, Y. Kwon, and Y. Fang. Performance analysis of IEEE 802.11 MAC protocols in wireless LANs. Wireless Communications and Mobile Computing, 4(8):917-931, 2004.

[22] L. Zhang, Y. Shu, and O.W.W. Yang. Throughput and fairness improvement in $802.11 \mathrm{~b}$ multi-rate WLANs. In Proceedings of the 16th IEEE International Symposium on Personal, Indoor and Mobile Radio Communications, volume 3, pages 1946-1950, 2005. 